\title{
Particle Swarm Extension to LOLIMOT
}

$\begin{array}{cccc}\text { Ramin Mehran } & \text { Alireza Fatehi } & \text { Caro Lucas } & \text { Babak Nadjar Araabi } \\ \text { K.N.Toosi University of } & \text { K.N.Toosi University } & \text { University of } & \text { University of } \\ \text { Technology } & \text { of Technology } & \text { Tehran } & \text { Tehran } \\ \text { RMehran@ee.kntu.ac.ir } & \text { Fatehi@kntu.ac.ir } & \text { Lucas@ipm.ir } & \text { Araabi@ut.ac.ir }\end{array}$

\begin{abstract}
In this paper, we will present population based method for placement of center of radial basis function of a locally linear neuro-fuzzy (LLNF) network, which is trained by LOLIMOT algorithm. Originally, LOLIMOT algorithm incrementally divides the hyperrectangles on input space into two axis orthogonal directions in half. However, this heuristic method would not be the best possible partitioning of the space. We present and evaluate a new Particle Swarm Optimization (PSO) method for finding the best divisions of input space in the LOLIMOT algorithm.
\end{abstract}

\section{Introduction}

A local linear modeling approach is based on a dived-and-conquer strategy. A complex modeling problem is divided into a number of smaller and thus simpler subproblems, which could be solved almost independently by simple models. The most important factor for the success of such an approach is the division strategy for the original complex problem [1]. Hence, the accuracy of LLNF models essentially depends on how the division strategy works. LOLIMOT algorithm provides a simple, fast, deterministic, and incremental division strategy, which has low number of trial-and-error steps for identification [2]. Although excluding the nonlinear optimization methods form LOLIMOT has increased its speed, the algorithm may produce redundant local models and lack the ability to find the global optimum structure. Thus, a key point for improving the performance of the LOLIMOT algorithm is to optimize its division strategy by a global search method.

Finding the best divisions in the LOLIMOT algorithm resembles to finding the center and variance of the Gaussian radial basis functions in an RBF network [3]. However, the LOLIMOT algorithm has a concrete method for making divisions and assigning locally linear model to them. The appealing idea to optimize this systematic strategy is to add some stochastic behavior to the creation of division and try to find the best local linear structure. Surely, this increases the number of calculations but it provides means for reduction in number of linear models and decrease in the overall error of the model.

Particle swarm optimization (PSO) is a population based stochastic optimization technique, originally developed by Kennedy and Eberhart in 1995 [4,5]. PSO has grown in the past several years extensively and compared with Genetic Algorithm (GA), PSO has benefits of memory and sharing of information among particles in the swarm [6,7, and 8].

In the field of neural networks, PSO has been applied to three main attributes of neural networks: network connection weights, network architecture (network topology, transfer function), and network learning algorithms. Most of the work involving the evolution of ANNs has focused on the network weights and topological structure [7]. Besides neural networks training, PSO has been combined with techniques to solve different problems such as fuzzy system [9], support vector machine [10], and recently locally linear wavelet networks [11]. In this paper, we will present a swarm extension to LOLIMOT algorithm, which utilizes PSO to find the best division strategy for locally linear models.

The following sections are organized as follows. The LLNF and LOLIMOT is shortly reviewed in section 2. In section 3, the PSO method, used for optimizing the division strategy of LOLIMOT, is discussed. Section 4 includes experimental results for the proposed swarm extension. Finally, concluding remarks are derived in the last section.

\section{LLNF and LOLIMOT}

The LLNF networks is depicted in Figure 1. Each neuron realizes a local linear model (LLM) and an 
associated validity function that determines the region of validity of the LLM [1]. The validity functions, which are similar to basis functions in RBF and could be Gaussians, are normalized such that $\sum^{M} \Phi_{i}(\underline{z})=1$ for

any input $z$. The output of this model is calculated as $\hat{y}=\sum_{i=1}^{M}\left(w_{i, 0}+w_{i, 1} x_{1}+\ldots+w_{i, n x} x_{n x}\right) \Phi_{i}(\underline{z})$

where the local linear models depend on $\underline{x}=\left[\begin{array}{llll}x_{1} & x_{2} & \ldots & x_{n x}\end{array}\right]^{T}$ and the validity functions depend on $\underline{z}=\left[\begin{array}{lllll}z_{1} & z_{2} & \ldots & z_{n x}\end{array}\right]^{T}$. However, we simply consider the case where the linear models and validity functions use the same inputs $x$. This network simply interpolates linear hyper-planes, which are used to approximate the functions locally, by nonlinear neurons called validity function.

A typical choice for validity function is normalized Gaussians, which are furthermore axis orthogonal.

$\Phi_{i}(\underline{z})=\frac{\mu_{i}(\underline{z})}{\sum_{j=1}^{M} \mu_{i}(\underline{z})} \quad$ where, $\mu_{i}(\underline{u})=\prod_{j=1}^{p} \exp \left(-\frac{1}{2} \frac{\left(u_{j}-c_{i j}\right)^{2}}{\sigma_{i j}^{2}}\right)$

The centers and standard deviations in (2) are nonlinear network parameters of (1). Each Gaussian function in (2) performs as a membership function with input vector $x$ for the locally linear model with and input vector $\mathrm{z}$ and $w_{i j}$ is the $j$-th local weight of the linear system $i$. The input space is decomposes in axis orthogonal manner yielding hyper-rectangles which centers of Gaussian membership functions $\mu_{i}(z)$ are placed. The standard division of these Gaussians is set to $1 / 3$ of the length of their rectangles in each dimension.

The LOLIMOT algorithm [2] simply asserts to split the hyper-rectangles in halve but there is no guarantee that we could not perform more effective strategy at this step. Figure 2 shows an example of weakness of simply taking half divisions in LOLIMOT algorithm in approximation of function

$$
z=\operatorname{sinc}(x, y)=\frac{\sin x}{x} \cdot \frac{\sin y}{y} \text { where }(\mathrm{x}, \mathrm{y}) \in[-10,10] \times[-10,10]
$$

\section{PSO Extension to LOLIMOT}

First, we will introduce PSO algorithm in brief and in the second part, we will provide combination of PSO and LOLIMOT.

\subsection{Particle Swarm Optimization}

The swarm is initialized with a group of random particles (solutions) and then searches for optima by updating each generation. In every iteration, each particle is updated by following two "best" values. The first one is the location of the best solution (fitness) a particle has achieved so far. This value is called pBest. Another "best" value is the location of the best solution that any neighbor of a particle has achieved so far. This best value is a neighborhood best and called $n B e s t$. When a particle takes all the population as its neighbors, the best location is a global best and is called $g$ Best. In this paper we have merely used global population and no local neighboring.

The core of the PSO is the updating formulae of the particle according to equations (4) and (5), respectively:

$$
\begin{aligned}
& v_{i d}=w \times v_{i d}+c_{1} \times \text { rand }() \times\left(p_{i d}-x_{i d}\right) \\
& +c_{2} \times \operatorname{Rand}() \times\left(p_{\text {gd }}-x_{i d}\right) \\
& x_{i d}=x_{i d}+v_{i d}
\end{aligned}
$$

Equation (4) calculates a new velocity for each particle (potential solution) based on its previous velocity $\left(v_{i d}\right)$, the particle's location at which the best fitness sofar has been achieved ( $p_{i d}$, or $\left.p B e s t\right)$, and global best location ( $\mathrm{p}_{\mathrm{gd}}$, or $\left.g B e s t\right)$ at which the best global fitness is acheved. Equation (5) updates each particle's position in the solution hyperspace. Rand() and $\operatorname{rand}()$ are two random numbers independently generated in the $[0,1]$ interval. $C_{1}$ and $C_{2}$ are two learning factors, which control the influence of $p B e s t$ and $g B$ Best on the search process. The use of inertia weight improves the global search performance [12]. [7] has a detailed explanation over the selecting proper PSO parameter along with a discussion about the importance of each.

\subsection{Combining PSO and LOLIMOT}

The most essential step to for applying PSO to any problem is changing the representation of the nonlinear problem to the algorithm framework. The next key issue is to define the individuals and objective function that ensures the optimization.

3.2.1. Defining a proper representation. Each LLNF network is considered as the individual in PSO algorithm and the objective function is the overall error of the model. The structure of the LOLIMOT model could be summarized in a matrix called map, which is a lower triangular matrix that represents the direction and position of divisions at each decomposition level incrementally. For sake of simplicity, we will discuss the formation of this map for 2-D case.

Every division involves cutting a hyper-rectangle in two axis orthogonal directions. We could represent these two direction by minus and plus signs. In multidimensional space, it will be a multi-value symbol. The location of division is defined by a ratio 
value, which is the ratio of length of vector $v$, to the length of the side of the rectangle that is parallel to the that vector. The vector $v$ is drawn from the minimum bound of the rectangle to the cut point perpendicular to the cut direction as shown in Figure 3. Thus, a single value and a sign could represent every division at each level of LOLIMOT.

Each $i=1 \ldots M$ row of the map matrix represents the $i$-th level of decomposition which have produced $i$ locally linear models (LLM). At each row, denoted by $i$, the $m$-th cell will contain non-zero element with value of ratio in it and the rest of the row are set to zero. This division adds a new row, $i+1$, to the matrix. The divided cell has become two cells in the new row, which are placed in the cell below the previous cell, $m$, and the neighboring cell to the right, $m+1$. At each division, the indices of LLMs, which are greater than $m$ at row $i$ will be increased by 1 and the rest of indices will be intact. Figure 4 shows the production of the map matrix for the original LOLIMOT algorithm after 5 divisions.

The map matrix puts the structure of the LOLIMOT algorithm in a mathematical representation suitable for PSO. Transition from row $i$ to $i+1$ produces a unique division line in a certain direction. The swarm individual is a solution structure, which is defined by division lines. We will select the position of each of these division lines as one dimension of our particle swarms. Thus, running the LOLIMOT at level $i$, with $i$ division lines, we could produce swarms with $i$ dimensions. Since we intend to optimize the structure of LOLIMOT at each level $i$, the optimization reduces to a search in an $i$ dimensional space.

We used the map representation in forming a swarm equivalent to the structure as it is depicted in Figure 5. This figure shows a modified LOLIMOT structure in level 4 of Figure 4 along with corresponding $1 \times 3$ swarm and $3 \times 3$ map matrix. It should be noted that since swarm of length $k$ represent $k$ division lines, it produces $k+1$ LLMs.

Therefore, changing the swarm values in each dimension equals to sliding the division lines in the LOLIMOT structure. In addition, the algorithm should consider a sliding interval for each division line to ensure that the structure does not collapse. This simple rule will assure this: "Every two division-line, which their projection involves more than two points, should not overlap or pass over each other." Figure 6 illustrates this constraint for two important cases. In this example, the red line and blue lines should not overlap or pass each other, but the blue line do not put any condition on the movement of each other since their projection have only one point in common. Meanwhile, the interval for sliding of division lines is defined as limited to the half way toward adjacent conditioning line and the boundary of the input space. This ensures that at each iteration of PSO algorithm, all swarm will be valid LOLIMOT structures.

To deal with the constraints, we limited the search space of PSO at each iteration to the possible solutions, which meet the mentioned conditions. Swarms, which try to pass the boundaries, are clamped to the boundary values. This method resembles to the implemented approaches of $[13,14]$, but it differs in some special factors that LOLIMOT has created.

3.2.2. Selected parameters of PSO and modifications to PSO and LOLIMOT. We will present the used PSO parameters in our experiments and meanwhile, we emphasis on the modifications we have done on the original PSO and LOLIMOT to combine them.

We have used constant value for $c_{1}$ and $c_{2}$ equal to 2 and linearly decreasing weight, $w$, in (4). The large values of weight at the beginning of the algorithm help the swarms to search globally and as the swarm converges at the end of iterations the small value of weight improves the local search abilities [12]. The two independent random values, $\operatorname{Rand}()$ and $\operatorname{rand}()$, are selected to have uniform distribution over $[0,1]$ same as the original PSO. The population number is set to constant 20 for simplicity of the algorithm.

We have developed two extensions to original LOLIMOT, which the first one is simpler, the second one is the natural generalization of the first, and we call the later fully swarmed LOLIMOT. In the first extension, the LOLIMOT algorithm is performed independently to produce $M+1$ LLMs at level $M$. Meanwhile, then the map matrix of corresponding LLM structure should be produced simultaneously with run of LOLIMOT. Using the map matrix, we produce a population of swarms of $M$ dimensions. The next step is to enter the swarms to a swam-pool, which iteratively evaluates the new position of swarms with respect to constraints. This swarm pool then create corresponding new LLMs for new position of the swarm. The calculation of output signal and model error for each LLM structure will be the same as LOLIMOT algorithm. At the end of each iteration, the best division of LOLIMOT algorithm is preserved in gBest and $p_{g d}$ of the PSO algorithm and the optimization continues until the error goal or maximum number of iterations is reached. Thus, summarized version our swarm extension to LOLIMOT is described in Appendix I.

To optimize the LOLIMOT structure at each level instead of optimization of its final structure, we made some considerations. Since the population-based algorithms are more time consuming, we decided to 
accept the LOLIMOT decision for direction of divisions and we only use our PSO algorithm in optimizing the location of the division line. This will roughly decrease the execution time of swarm extension of the LOLIMOT to half, but it may create suboptimal solution instead. Nevertheless, we could simply avoid this decision of LOLIMOT and try to find the direction of the division at together with location of it in the swarm pool when global optimality is necessary.

In addition, some modification has to be done on the original PSO to preserve the best solution of LOLIMOT of level $i$ for the next level. Thus, the initial population should contain the swarm of the best division selected by LOLIMOT at every stage. Meanwhile, to preserve the structure of the LLM structures and to limit the search space the algorithm should regard the old line-division and should pay more attention to the newly created ones. This property resembles the forgetting factor in online system identification and memory reset strategy in PSO [15]. To implement this forgetting behavior we decided to weight the calculated velocity of PSO in (4) by an exponential decay factor,

$v_{i d}=v_{i d} *$ decay $\quad$, where decay $y_{i}=\exp (-i / 20)$

By introducing the decay term in (6) and step 2 of the LOLIMOT algorithm, the changes in the swarm position in the problem space is force to be carried out on the high energy section of the space which are responsible for creating errors by most. Hence, our proposed strategy for preserving the LOLIMOT structure, protects the LOLIMOT's appealing property, that decomposes the section of the space with more data point [2]. To sum up, the fully swarmed LOLIMOT algorithm is provided in Appendix II

\section{Implementation and Experimental Results}

In this section, we will provide the results of our experiments on approximation of the function (3) with our proposed method. We have trained an LLNF for (3) and by cross validation methods we concluded that $\mathrm{M}=42$ would be a good approximation of the efficient number of LLMs in LOLIMOT method. Here, we will compare those results with the results form our PSO extension to LOLIMOT. In this implementations we will mention our first method as PSO extension of LOLIMOT and the second method as fully swarmed LOLIMOT.

For estimation of output weights of the LLMs we have used the train set data, which has 121 data points and for the validation and test we have used the 71 points of the test set. The test and train sets were created randomly with uniform distribution of the domain of (3). It should be noted that for preserving the property of generalization of the approximations, the test set was used for calculation of the objective function in PSO algorithms, but the training of the LLM were always employed by train set. In this section, all of the error calculations is reported on the test set data.

Figure 7 and Figure 8 show the result for the PSO extension of LOLIMOT on the test in different conditions. It is shown that with the same number of LLMs our method is able to achieve lower error rates comparing to the simple LOLIMOT. In addition, Figure 8-Right shows that the PSO extension of LILIMOT has achieved better results than original LOLIMOT even by consuming far less LLMs. This behavior has been expected, since the algorithm is designed to find the optimum structure of LLMs. The results also indicate that the using the decay weighting in PSO extension of LOLIMOT does not provide appropriate results, since the LOLIMOT strategy of half divisions creates suboptimal structures.

On the other hand, the fully swarmed LOLIMOT method provides even better results at the cost of more computations. Figure 9 shows the results of each algorithm when $\mathrm{M}=42$ and $\mathrm{M}=30$. By comparing the results with the original LOLIMOT, it is clear that our method provides more promising results with considerably less error on the test set. Table 1 summarized the comparisons of the different method.

\section{Summary}

In this paper, we have presented a swarm extension to LOLIMOT training algorithm of LLNF networks, which to the best of our knowledge, no similar works was reported in the literature. The development of the method includes creation of a flexible representation for LOLIMOT decomposition strategy. In addition, we have made modification to original PSO algorithm to combine it with LOLIMOT. The implementation results of our algorithms show considerable improvement over LOLIMOT and emphasis that with this optimization technique we are able to produce more accurate LLNF model with less locally linear models.

\section{References}

[1] O. Nelles, Nonlinear system identification: from classical approaches to neural network and fuzzy models. Berlin, Germany: Springer-Verlag, 2001. 
[2] O. Nelles, A. Fink, R. Isermann, Local Linear Model Trees (LOLIMOT) Toolbox for Nonlinear System Identification, 12th IFAC Symposium on System Identification (SYSID), Santa Barbara, USA, 2000.

[3] VM. Rivas, PA. Castillo, JJ. Merelo."Evolving RBF neural networks. Connectionist Models of Neurons, Learning Processes, and Artificial Intelligence". 6th International Work-Conference on Artificial and Natural Neural Networks, IWANN 2001. Proceedings, Part I (Lecture Notes in Computer Science Vol. 2084). Springer-Verlag. 2001, pp.506-13. Berlin, Germany.

[4] J. Kennedy, and R. Eberhart, "Particle swarm optimization.", Proceedings of IEEE international Conference on Neural Networks, pp. 1942-1948,1995

[5] Y. Shi, R. Eberhart, "A modified particle swarm optimizer", IEEE International Conference on Evolutionary Computation Proceedings. IEEE World Congress on Computational Intelligence, pp.69-73. New York, NY, USA 1998.

[6] Chia-Feng Juang. "A hybrid of genetic algorithm and particle swarm optimization for recurrent network design", IEEE Transactions on Systems, Man, \& Cybernetics, Part B: Cybernetics, vol.34, no.2, , pp.997-1006, USA, April 2004.

[7] H. Xiaohui, Y. Shi, R. Eberhart "Recent advances in particle swarm", Proceedings of the 2004 Congress on Evolutionary Computation, 2004.

[8] R. Eberhart, and Y. Shi, "Particle Swarm Optimization: Developments", Applications and Resources,Proceedings of the IEEE Congress on Evolutionary Computation (CEC 2001), Seoul, Korea. 2001

[9] Z. He, C. Wei, L.Yang, X. Gao, S. Yao, R. Eberhart, and Y. Shi, "Extracting rules from fuzzy neural network by particle swarm optimization", Proc. IEEE International Conference on Evolutionary Computation, Anchorage, Alaska, USA, 1998.

[10] U. Paquet, and AP. Engelbrecht, "Training support vector machines with particle swarms". Proceedings of the International Joint Conference on Neural Networks, pp.1593-8 vol.2, 2003.

[11] 1. Yuehui Chen, Jiwen Dong, Bo Yang, Yong Zhang, "A local linear wavelet neural network", Fifth World Congress on Intelligent Control and Automation., pp.1954-7 Vol.3., 2004.

[12] Cui Zhihua, Zeng Jianchao, Cai Xingjuan. "A new stochastic particle swarm optimizer", Proceedings of the 2004 Congress on Evolutionary Computation pp.316-19 Vol.1, 2004

[13] AI . El-Gallad, ME. El-Hawary, AA. Sallam "Swarming of intelligent particles for solving the nonlinear constrained optimization problem", International Journal of Engineering Intelligent Systems for Electrical Engineering \& Communications, vol.9, no.3, pp.155-63. Publisher: CRL Publishing, UK, Sept. 2001.
[14] X. Hu, and R. Eberhart, "Solving constrained nonlinear optimization problems with particle swarm optimization", 6th World Multiconference on Systemics, Cybernetics and Informatics (SCI 2002), Orlando, USA, 2002.

[15] A. Carlisle, and G. Dozier, "Adapting particle swarm optimization to dynamic environments", Proceedings of the International Conference on Artificial Intelligence. ICAI'2000. CSREA Press. Part vol.1, p p.429-33 vol.1. Athens, GA, 2000

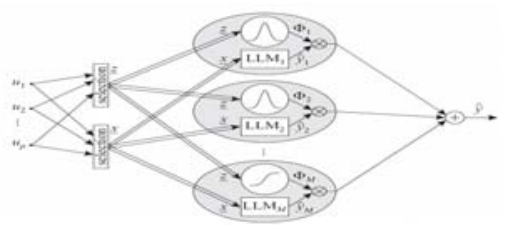

Figure 1. Locally Linear Neuro-Fuzzy Network
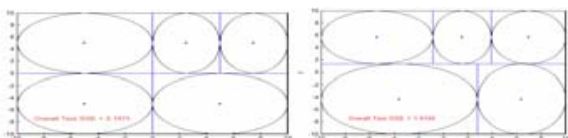

Figure 2. Approximation of function (3) (Left) Orginal LOLIMOT with half division strategy SSE $=2.19$ (Right) Not taking half division in LOLIMOT SSE $=1.91$

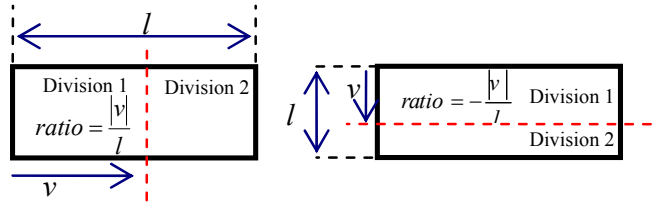

Figure 3. Definition of ratio in the decomposition strategy of (hyper-)rectangles

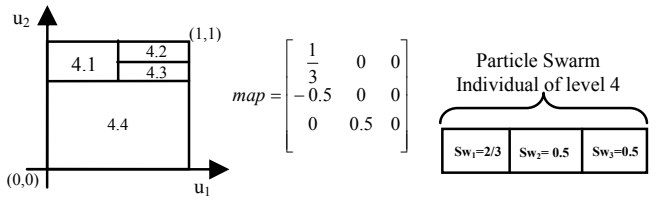

Figure 4. Example of LOLIMOT structure, equivalent map matrix, and swarm vector 


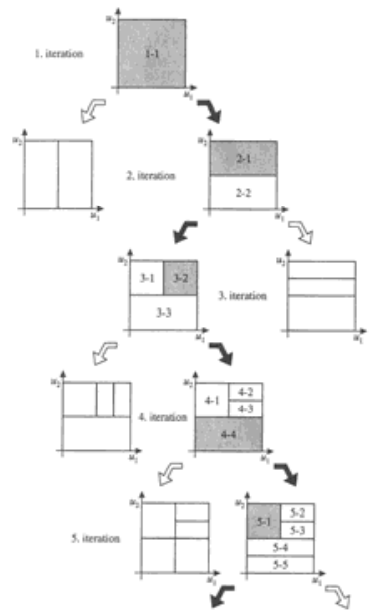

map $=[0.5]$

map $=\left[\begin{array}{cc}0.5 & 0 \\ -0.5 & 0\end{array}\right]$

map $=\left[\begin{array}{ccc}0.5 & 0 & 0 \\ -0.5 & 0 & 0 \\ 0 & 0.5 & 0\end{array}\right]$

$\left[\begin{array}{cccc}0.5 & 0 & 0 & 0 \\ -0.5 & 0 & 0 & 0 \\ 0 & 0.5 & 0 & 0\end{array}\right]$

map $=\left[\begin{array}{llll}0.5 & 0 & 0 & 0 \\ 0 & 0.5 & 0 & 0\end{array}\right.$

$\begin{array}{llll}0 & 0 & 0 & 0.5\end{array}$

$0.5 \quad 0 \quad 0 \quad 000$

$-0.5 \quad 0 \quad 0000$

map $=\mid \begin{array}{lllll}0 & 0.5 & 0 & 0 & 0\end{array}$

$\begin{array}{lllll}0 & 0 & 0 & 0.5 & 0 \\ -0.5 & 0 & 0 & 0 & 0\end{array}$

Figure 5. Operation of LOLIMOT search structure (courtesy of [1]) and our equivalent map matrix for 5 division. (shaded block and black arrows represents selected blocks and divisions respectively)

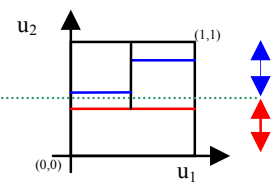

Figure 6. The valid interval for sliding the division lines in LOLIMOT structure.

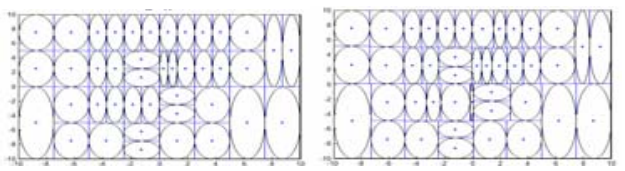

Figure 7. LOLIMOT Structure, (Left) The original LOLIMOT structure for (3) with $\mathrm{M}=42 \mathrm{MSE}=0.0017,(\mathrm{R}$ ight) The first method of PSO extension to LOLIMOT wi th $\mathrm{M}=42$ and decay weighting $\mathrm{MSE}=0.0014$ Max Iteration $=100$

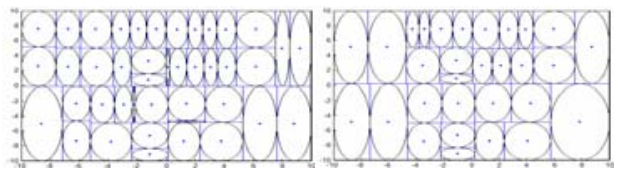

Figure 8. PSO extension of LOLIMOT structure, with no decay weightings, Maximum iterations $=100$ (Left) $\mathrm{M}=$ $42 \mathrm{MSE}=9.21 \mathrm{e}-4$ (Right) $\mathrm{M}=30 \mathrm{MSE}=0.0012$

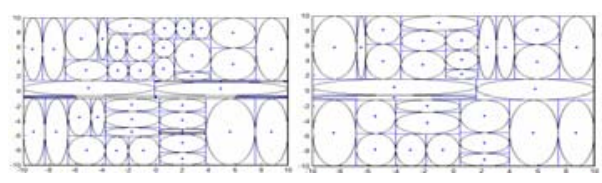

Figure 9. Fully swarmed LOLIMOT, Maximum Iteration at each level of LOLIMOT $=20$, with decay weighting,

(Left) $M=42, M S E=8.19 \mathrm{e}-4$

(Right) $M=30, M S E=9.93 e-4$
Appendix I

1. Run LOLIMOT algorithm to produce $M$ division lines and create the corresponding
map matrix simultaneously.
2. Create a random initial population of swarms based on the map matrix in step 1.
3. Enter the swarms to the swarm pool
4. Do
4.1.Change swarm values according to PSO with respect to constraints
4.2.Calculate the corresponding map and LLM structure for each swarm (includes
least square estimation of output weight of LLNF and updating of all validity
functions).
4.3.Evaluate each swarm by calculation of error of the corresponding LLMS.
4.4.Use the resultant errors for PSO evaluation
5. Until the error goal or maximum number of PSO iterations is reached.

Table 1. Comparison of our two methods of PSO extension to LOLIMOT in different conditions and LLNF traning of (1) Original LOLIMOT, (2) to (4) PSO extension 1 to $3,(5)$ and (6) Fully swarmed 1 and 2

\begin{tabular}{|c|c|c|c|}
\hline & M & $\begin{array}{c}\text { MSE on } \\
\text { test set }\end{array}$ & Conditions \\
\hline 1 & 42 & $17 \mathrm{e}-4$ & \\
\hline 2 & 42 & $14 \mathrm{e}-4$ & $\begin{array}{c}\text { Max iterations }=100, \\
\text { with decay weighting }\end{array}$ \\
\hline 3 & 42 & $9.21 \mathrm{e}-4$ & $\begin{array}{c}\text { Max iterations }=100, \\
\text { no decay weighting }\end{array}$ \\
\hline 4 & 30 & $12 \mathrm{e}-4$ & $\begin{array}{c}\text { Max iterations }=100, \\
\text { no decay weighing }\end{array}$ \\
\hline 5 & 42 & $8.19 \mathrm{e}-4$ & $\begin{array}{c}\text { Max iterations }=20 \text { at } \\
\text { each level of } \\
\text { decomposition, with } \\
\text { decay weighting }\end{array}$ \\
\hline 6 & 30 & $9.93 \mathrm{e}-4$ & $\begin{array}{c}\text { Max iterations }=20 \text { at } \\
\text { each level of } \\
\text { decomposition, with } \\
\text { decay weighting }\end{array}$ \\
\hline
\end{tabular}

\section{Appendix II}

1. Start with an initial model: Construct the validity functions for the
initially given input space partitioning and estimate the LLM
parameters by the local weighted least squares algorithm. Create the
initial map matrix.
Find worst LLM: Calculate a local loss function for each of LLMs.
Find the worst performing LLM, and denote $l$ as the index it.
3. $\begin{aligned} & \text { Check all divisions: The LLM } l \text { is considered for further refinement. } \\ & \text { The hyper-rectangle of this LLM is split into two halves with an axis } \\ & \text { orthogonal split. Divisions in each dimension are tried. }\end{aligned}$
Find best division: The best of the all alternatives checked in Step 3 is
selected. Update the map matrix with the new division.
Create initial population: Create an initial population of swarms based
on the map matrix which includes the best LLM till now.
Calculate the decay weights: use equation (6) for creating decay
weights.
Enter the swarms to the swarm pool: Create map for each swarm.
Do
Do Change swarm values according to PSO with respect to constraints
8.1 Calculate the corresponding map and LLM structure for each
8.2
swarm (includes least square estimation of output weight of LLNF
and updating of all validity functions).
8.3 Evaluate each swarm by calculation of error of the corresponding
LLMS.
8.4 Use the resultant errors for PSO evaluation
Until the error goal or maximum number of PSO iterations is reached.
10. Test for convergence: If the termination criterion is met then
stop else go to step 2.

